\title{
Chemical peritonitis associated with intraperitoneal vancomycin: A case series
}

\author{
José Silvano ${ }^{1,2}$, Luciano Pereira ${ }^{1,2}$, Ana Oliveira ${ }^{1,2}$, Ana Beco ${ }^{1,2}$, Miguel Relvas ${ }^{1,2}$, Rui Silva ${ }^{1,2}$, Manuel Pestana ${ }^{1,2,3}$ \\ ${ }^{1}$ Nephrology department, Centro Hospitalar e Universitário da São João. Porto, Portugal \\ ${ }^{2}$ Nephrology \& Infectious Diseases R\&D, i3S - Instituto de Investigação e Inovação em Saúde da Universidade do Porto, Porto, Portugal \\ ${ }^{3}$ Faculty of Medicine, University of Oporto, Porto, Portugal
}

\section{ABSTRACT}

Peritonitis is a serious complication in peritoneal dialysis, usually secondary to an infectious cause. Chemical peritonitis is rarer. No case exclusively attributed to vancomycin has been reported in the last 20 years. Data from 4 consecutive patients diagnosed with culture-negative peritonitis following administration of intraperitoneal vancomycin between May and June 2019 were retrospectively recorded. All patients were treated with 2 grams of intraperitoneal vancomycin after a break in aseptic technique and developed a cloudy effluent. No patient was previously known to be allergic to vancomycin. All had a clear dialysate before vancomycin. All developed an elevated leukocyte count in the dialysate. All had sterile cultures. All resumed a clear effluent with less $<100$ cells $/ \mu \mathrm{L}$ after vancomycin cessation, and in two there were no further administrations. In one, a new drug challenge led to recrudescence of abdominal pain and reappearance of a cloudy sterile effluent. In another, vancomycin from a different lot was administrated 3 days after, no symptoms developed and dialysate cell count remained normal. The pathogenic mechanisms underlying chemical peritonitis are not fully known. The clinical course is typically benign. Management seems to be limited to drug withdrawal. If unrecognized, chemical peritonitis may ultimately lead to unnecessary catheter removal.

Keywords: Chemical peritonitis; peritoneal dialysis; vancomycin.

(C) 2021 Portuguese Journal of Nephrology \& Hypertension. Published by Publicações Ciência \& Vida This is an open access article under the CC BY-NC-ND license (http://creativecommons.org/licenses/by-nc-nd/4.0/).

\section{INTRODUCTION}

Peritonitis is one of the most frequent and serious complications in patients undergoing peritoneal dialysis (PD), often leading to catheter loss, conversion to long-term hemodialysis and death in $>5 \%$ of the cases $^{1,2}$. It is usually secondary to an infectious cause (pathogenic bacteria and fungi) and as such, patients presenting with a cloudy effluent should be treated presumptively for infectious peritonitis until the diagnosis can be confirmed or excluded ${ }^{3}$. Intraperitoneal (IP) vancomycin has been widely advocated as the first-line empiric therapy in many PD units, due to the high rate of methicillin-resistant organisms ${ }^{4}$.

Peritonitis with negative effluent cultures can sometimes be attributed to an inadequate culture technique after inoculation in blood-culture bottles, which accounts for about $10-20 \%$ of cases ${ }^{5}$. A rarer cause is chemical peritonitis with sterile cultures, following exposure to non-infectious agents such as amphotericin B or an antibiotic, dialysis preparations and compounds.

We report a series of cases of chemical peritonitis with sterile cultures, in which vancomycin seems to be the causative agent (Table I).

\section{METHODS}

Clinical and demographic characteristics of patients diagnosed with culture-negative peritonitis following empiric administration of IP vancomycin in our institution between May and June 2019 were retrospectively recorded.

Peritonitis was defined as the presence of 2 of the following: suggestive clinical features, positive effluent culture, dialysis effluent white cell count $>100 / \mu \mathrm{L}$ or $>0.1 \times 10^{9} / \mathrm{L}$ (after a dwell time of at least 2 hours), with > $50 \%$ polymorphonuclear (PMN) cells ${ }^{3}$. When present, we start empiric IP antibiotic therapy covering both gram negative (ceftazidime 1 gram IP, daily) and methicillin-resistant gram positive (vancomycin 2 grams IP on the first two days, and subsequent doses according to serum levels).

Two grams of IP vancomycin are also administrated after a break in aseptic technique, usually as a single dose in the following PD exchange, even in the absence of a cloudy effluent.

\section{CASE REPORTS}

Four consecutive prevalent patients on a regular PD program, treated with 2 grams of IP vancomycin (Vancomycin Hikma) from the same lot as prophylaxis following a break in aseptic technique, developed a cloudy effluent after the exchange to which the drug was added.

Patient 1: 67 year-old female on PD for 2 years due to end-stage renal disease (ESRD) secondary to autosomal dominant polycystic kidney disease (ADPKD). 


\section{Table I}

Patients clinical evolution after vancomycin administration

\begin{tabular}{|c|c|c|c|c|c|c|c|c|}
\hline $\begin{array}{l}\text { Patient } \\
\text { number }\end{array}$ & $\begin{array}{c}\text { Reason for Vancomycin } \\
\text { administration }\end{array}$ & $\begin{array}{c}\text { Body weight (BW) } \\
\text { and dose administrated }\end{array}$ & $\begin{array}{c}\text { Time to cloudy } \\
\text { dialysate }\end{array}$ & Clinical features & $\begin{array}{c}\text { Dialysate white } \\
\text { blood cells (\% PMN) }\end{array}$ & $\begin{array}{l}\text { Effluent } \\
\text { cultures }\end{array}$ & $\begin{array}{c}\text { Positive rechallenge } \\
\text { test }\end{array}$ & $\begin{array}{l}\text { Vancomycin } \\
\text { from a new lot }\end{array}$ \\
\hline 1 & $\begin{array}{l}\text { break in the aseptic } \\
\text { technique }\end{array}$ & $\begin{array}{l}\text { BW: } 60.2 \mathrm{~kg} \\
\text { Dose: } 2 \text { grams }\end{array}$ & $<24 h$ & $\begin{array}{l}\text { Cloudy effluent, } \\
\text { abdominal pain on } \\
\text { deep palpation }\end{array}$ & 5767 (PMN 73.6\%) & Negative & No & No \\
\hline 2 & $\begin{array}{l}\text { break in the aseptic } \\
\text { technique }\end{array}$ & $\begin{array}{c}\text { BW: } 72 \mathrm{~kg} \\
\text { Dose: } 2 \text { grams }\end{array}$ & $\begin{array}{c}\text { 4h (after } \\
\text { vancomycin } \\
\text { exchange) }\end{array}$ & Asymptomatic & 115 (PMN 22\%) & Negative & No & No \\
\hline 3 & $\begin{array}{c}\text { break in the aseptic } \\
\text { technique }\end{array}$ & $\begin{array}{c}\text { BW: } 57 \text { kg } \\
\text { Dose: } 2 \text { grams }\end{array}$ & $<24 h$ & $\begin{array}{c}\text { Abdominal pain, itching } \\
\text { and cloudy effluent }\end{array}$ & 8825 (PMN 84.5\%) & Negative & $\begin{array}{l}\text { Yes (abdominal pain } \\
\text { + cloudy effluent) }\end{array}$ & No \\
\hline 4 & $\begin{array}{c}\text { break in the aseptic } \\
\text { technique }\end{array}$ & $\begin{array}{c}\text { BW: } 72 \text { kg } \\
\text { Dose: } 2 \text { grams }\end{array}$ & $<24 h$ & Abdominal pain & 2032 (PMN 40\%) & Negative & $\begin{array}{l}\text { Yes (dialysate } \\
\text { with } 1472 \text { cells } \\
\text { and } 40 \% \text { PMN) }\end{array}$ & $\begin{array}{l}\text { Yes (clear effluent } \\
+ \text { absence of } \\
\text { symptoms) }\end{array}$ \\
\hline
\end{tabular}

On day 1 , she went to the hospital after noticing an effluent leak from the catheter exit-site starting 3 days prior. There were no inflammatory signs or purulent discharge from the exit-site There was no loss of integrity of the catheter, but the extension of the catheter was substituted. She was asymptomatic. Dialysate leukocyte count was 17 cells/ $\mu \mathrm{L}$ (88\% PMN), effluent cultures were drawn and IP vancomycin (2 grams) was started. She came back the next day afebrile, but with a cloudy effluent, complaining of abdominal pain on deep palpation, anorexia and nausea. Dialysate leukocyte count was now of $5767 / \mu \mathrm{L}(73.6 \% \mathrm{PMN})$, and effluent cultures were once again collected. There was no leukocytosis, eosinophilia or significant C-reactive protein (CRP) elevation. Abdominal ultrasound was normal. A second dose of vancomycin was administrated together with ceftazidime (1g daily). On day 4, 48 hours after the last vancomycin administration, dialysate cell count was $56 / \mu \mathrm{L}$ (7.7\% PMN). A third set of effluent cultures was collected and a third dose of vancomycin of $500 \mathrm{mg}$ was administrated according to blood levels. She remained asymptomatic, and by day 8 all 3 effluent cultures had came back negative.

Considering the suggestive temporal association, a drug-reaction was suspected and no further doses of vancomycin were administrated. Dialysate leukocyte count remained $100 / \mu \mathrm{L}$, with a clear effluent and no more symptoms arising.

Patient 2: 64 year-old male on PD for 8 months with ESRD secondary to ADPKD. Previous history included a cloudy effluent secondary to lercanidipine ( 5 months prior) and an episode of infectious peritonitis treated with ceftazidime and vancomycin (Vancomycin Hikma) (completed 1 month prior, with Escherichia coli, Klebsiella oxytoca and Streptococcus spp. isolated in the effluent).

On day 1, a break in aseptic technique was documented (a leak in the dialysate solution bag), and vancomycin was administered as per protocol. Dialysate leukocyte count was $7 / \mu \mathrm{L}$ and effluent cultures were collected. The patient referred the development of a cloudy effluent after draining the antibiotic exchange. On day 2 he came back, already with a clear effluent and was asymptomatic. Dialysate leukocyte count was of 80/ $\mu \mathrm{L}$ (PMN 25\%). There was neither leukocytosis nor significant C-reactive protein (CRP) elevation. A new set of effluent cultures was drawn, coming back negative, as did the first. No more antibiotics were administrated and the effluent remained clear.

Patient 3: 53 year-old female on PD for 3 weeks with ESRD secondary to ADPKD.

On day 1, also after a break in aseptic technique (a leak in the dialysate solution bag), a dialysate leukocyte count of $22 / \mu \mathrm{L}$ was measured and effluent cultures were collected, after which IP vancomycin (2 grams) was administrated. She came back the next day complaining of diffuse abdominal pain, generalized itching without rash or skin lesions and a cloudy effluent. There was no peripheral leukocytosis nor eosinophilia. Peritonitis was assumed, and she was admitted for treatment in the nephrology ward. Dialysate leukocyte count was now of $8825 / \mu \mathrm{L}(84,5 \%$ PMN), a second set of cultures was collected, a second dose of vancomycin was administered together with ceftazidime (1 gram daily). On day 4, 48 hours after the last administration of vancomycin, dialysate leukocyte count was $384 / \mu \mathrm{L}(20.4 \% \mathrm{~N})$. On day 5 , vancomycin from the same lot was once again administrated according to blood levels, resulting in immediate and intense abdominal pain, accompanied by a burning sensation. Dialysate leukocyte count on day 6 was 15597/ $\mu \mathrm{L}$ (81\% PMN, 8 eosinophils). No further IP antibiotics were administrated, and both sets of effluent cultures came back negative. She remained asymptomatic afterwards, with a dialysate cell count of $466 / \mu \mathrm{L}$ on day 7 , negative on day 13 and a persistently clear effluent.

Patient 4: 55 year-old man on PD for 6 months with ESRD secondary to IgA nephropathy.

On day 1, a break in aseptic technique was documented (a leak in dialysis tubing while performing an exchange). Leukocyte count was 17 , effluent cultures were drawn and IP vancomycin was administrated (2 grams). He came back on day 2 with a cloudy effluent, diarrhea and abdominal pain on deep palpation. Dialysate leukocyte count was now 2032/ $\mathrm{LL}$ (40\% PMN, 46 eosinophils), and a new set of effluent cultures was drawn. No CRP elevation or leukocytosis was documented. A second IP dose of vancomycin was administrated together with ceftazidime (1 gram daily). On day 3 , dialysate contained a leukocyte count of $1472 / \mu \mathrm{L}$ (40\% PMN, $60 \%$ mononuclear cells). Both sets of effluent cultures came back negative. 
On day 6, he presented with a progressively less cloudy effluent, and a third dose of vancomycin from a different lot of the same brand was administrated. This did not elicit any recrudescence of the abdominal pain nor a cloudy effluent. No further vancomycin doses were administrated and he remained asymptomatic with a clear effluent. Follow-up dialysate leukocyte count was of $54 / \mu \mathrm{L}$ by day 10 .

\section{DISCUSSION}

Piraino and colleagues made the first observation of chemical peritonitis after IP vancomycin administration for catheter infection in $1986^{6}$. Near 90 cases were diagnosed in the 1980s and 1990 s, usually following loading doses $7,8,10$. One other isolated case of chemical peritonitis in a patient treated with icodextrin and IP vancomycin was reported in $2011^{11}$ (Table II).

In our series of 4 patients with chemical peritonitis induced by vancomycin, the unifying picture was the presence of a clear dialysate with a cell count $<100 / \mu \mathrm{L}$ and sterile effluent cultures before IP drugs were administrated (see Table I). All developed a de novo elevated leukocyte count (range 112 to $8825 / \mu \mathrm{L}$ ) after first drug administration. In all cases clinical course was benign, and peritonitis resolved with no further therapy. Time course from vancomycin IP administration to symptoms onset was very similar between patients and consistent with delivery of a chemical irritant to the peritoneal cavity. None of the patients was previously known to be allergic to vancomycin. Patient 2 had been given IP vancomycin before without any adverse reaction, and the remaining patients were never given IP vancomycin before.

The same vancomycin lot was used in all patients. During the same time period, 7 other patients received IP vancomycin in our center for the treatment of peritonitis, without any adverse event report. It was not possible to confirm if the same lot of vancomycin was given in all of them. If this was the case, a putative chemical reaction to vancomycin could have been masked due to concomitant bacterial peritonitis.

One cannot help but notice that 3 out of 4 patients had ADPKD as the etiology of ESRD, but we believe this is explained by the relatively high number of ADPKD patients in our program (20\%).
All cases were reported to INFARMED - National Authority of Medicines and Health Products, I.P. After switching to a new vancomycin lot, no further cases have been identified in our institution so far.

Certain features of the individual cases further support the diagnosis: in patient number 3 , a positive drug rechallenge was observed 3 days after, leading to recrudescence of abdominal pain and reappearance of a cloudy effluent, again with sterile cultures. This patient scored $>=9$ (definite) in the Naranjo adverse drug reaction probability scale (an adverse drug reaction score consisting of 10 questions to define probability with the reaction considered definite if the score is 9 or higher, probable if 5 to 8 , possible if 1 to 4 , and doubtful if 0 or less) ${ }^{12}$. In patient number 4 , vancomycin administration from a different lot was performed, 3 days after the first administration, after which no abdominal pain developed, and the dialysate cell count was $57 / \mu \mathrm{L}$. These two facts strongly suggest that IP vancomycin from the original lot caused chemical peritonitis, and that the inflammatory reaction could have been caused by a constituent of the preparation, rather than by vancomycin itself.

Clinical presentation is variable (mild, only with cloudy effluent, or more severe, with abdominal pain and fever), as was observed in our cases $^{8,9}$. In three of them, the cloudy effluent was accompanied by abdominal pain, and in one of them it was also accompanied by itching.

There was no leukocytosis, eosinophilia or significant CPR elevation in all. Differential counts of dialysate revealed a predominance of PMN cells in 3 cases, ranging from $22-88 \%$ in our series. This percentage is somewhat similar to the $45-84 \%$ range originally described by Piraino ${ }^{6}$. Eosinophil cell counts were available for 2 patients, and were $<2 \%$ in both, which is line with the $0-10 \%$ range of previous reports ${ }^{13,14}$.

Reactions are acute, usually taking 2-12 hours $^{12}$, and always $<24$ hours in our series, after the first loading dose exchange. We did not observe peripheral eosinophilia, although two cases of vancomycin-induced eosinophilic peritonitis have been previously reported ${ }^{15,16}$.

Evolution is typically benign. Management seems to be limited to drug withdrawal, with spontaneous resolution of signs and symptoms. The clinical picture typically resolves 3-4 days after suspension ${ }^{13}$ or until a new IP vancomycin dose was given, although long-term outcomes are unknown.

\section{Table II}

Review of the literature regarding chemical peritonitis following intraperitoneal vancomycin administration

\begin{tabular}{|c|c|c|c|c|}
\hline Cases & $\mathrm{n}$ & Positive rechallenge & Catheter removal & Death \\
\hline \multicolumn{5}{|l|}{ Case series } \\
\hline Piraino B et al, 1986 & 6 & $2 / 6$ & $2 / 6$ & 0 \\
\hline Charney D et al, 1991 & 4 & $1 / 4$ & 0 & 0 \\
\hline Freiman JP et al, 1992 & 51 & $9 / 51$ & 2 & 0 \\
\hline Wong PN et al, 1997 & 7 & $1 / 2$ & 0 & 0 \\
\hline \multicolumn{5}{|l|}{ Case reports } \\
\hline Smith T et al, 1991 & 1 & 1 & 0 & 0 \\
\hline $\begin{array}{l}\text { Freitas C et al, } 2011 \\
\text { (icodextrin + intraperitoneal vancomycin) }\end{array}$ & 1 & 1 & 1 & 0 \\
\hline
\end{tabular}


One case led to hospitalization and prolongation of the antibiotic treatment. Fortunately, vancomycin was eventually recognized as the inciting agent. However, as observed in previous case reports, a persistently cloudy effluent despite treatment with an antibiotic active against methicillin-resistant microorganisms may lead to presumption of a refractory infectious peritonitis. This can lead to inappropriate treatment and removal of an uninfected catheter, when an adverse reaction is not suspected ${ }^{6,8}$.

Finally, a cloudy effluent could have been caused by other factors such as a concurrent bacterial infection (unlikely regarding the repeatedly negative effluent cultures and the clinical resolution upon discontinuation of vancomycin), a chemical reaction to another substance (for example, icodextrin), calcium channel blockers, hemoperitoneum, malignancy or a chylous effluent ${ }^{3,17}$ but none of these provided an alternative explanation in our cases.

The clinical picture is less straightforward when vancomycin is administered for a confirmed diagnosis of infectious peritonitis, and a differential diagnosis with refractory peritonitis would be necessary in this case. Some clues are useful in this distinction. First, the absence of a positive culture, even though possible with infectious peritonitis, favors the hypothesis of a non-infectious etiology. More challenging is the case of an infectious peritonitis with a positive effluent culture, which does not improve with antibiotics. In this circumstance, the temporal association of signs of peritonitis with vancomycin administration is the main clue. These signs should disappear completely with drug withdrawal. If doubt remains, a drug challenge with intraperitoneal can be elicited to test if it clearly elicits signs and symptoms, which should follow after 2-12 hours and are more likely with higher doses ( 1 to 2 grams, as opposed to only $500 \mathrm{mg})^{12}$. The differential diagnosis is very important, as a misdiagnosis of refractory peritonitis may lead to unnecessary catheter removal and transition to hemodialysis.

The underlying pathogenic mechanisms of chemical peritonitis are not entirely understood. Previous reports of vancomycin-induced chemical peritonitis following loading doses, and with certain lots, suggest a local irritant effect related to the route of administration and concentration of IP vancomycin. Vancomycin is a biological product and therefore some degree of variability can be present between lots. The absence of a significant involvement of eosinophils and the association with specific lots, argue against an allergic mechanism.

The reaction could be caused by impurities in the vancomycin preparation or by vancomycin itself. This may be less common with newer and purer vancomycin solutions (6-11\% of total impurities were present in six different parenteral vancomycin preparations in a product quality analysis made in 2012, as opposed to larger amount in earlier preparations). Different quantities in individual lots may account for the varying intensity of the inflammatory reaction. High-performance liquid chromatography testing of the vancomycin lot was not possible in our cases but is indicated where available ${ }^{18}$. This would allow us to precisely quantify the impurities in the vancomycin preparation, and this way asses if an elevated percentage was a contributing cause.

This is the first series of cases of chemical peritonitis secondary to vancomycin reported in the last decade. True incidence could be underestimated, as some episodes could be masked by concomitant true bacterial peritonitis.

\section{CONCLUSION}

Our series of chemical peritonitis following vancomycin showed significantly heterogeneous reactions (from cloudy dialysate only, to abdominal pain). No fatalities were reported.

The absence of an infectious etiology for peritonitis, as well as the strongly suggestive temporal relation to the IP administration of the same lot of vancomycin brought to light the diagnosis of chemical peritonitis. It is unclear which specific mechanism elicited the drug reaction.

Despite this entity relative rarity, clinical awareness for the diagnosis is still fundamental - if unrecognized, chemical peritonitis may be presumed to be infectious, ultimately leading to potential catheter removal.

Disclosure of potential conflicts of interest: none declared.

\section{References}

1. Ghali JR, Bannister KM, Brown FG, Rosman JB, Wiggins KJ, Johnson DW et al. Microbiology and outcomes of peritonitis in Australian peritoneal dialysis patients. Perit Dial Int 2011; 31:651-62

2. Pérez-Fontán $M$, Rodríguez-Carmona $A$, García-Naveiro $R$, Rosales $M$, Villaverde $P$, Valdés $F$. Peritonitis-related mortality in patients undergoing chronic peritoneal dialysis. Perit Dial Int 2005;25(3):274-84

3. Li PK, Szeto CC, Piraino B, Arteaga J de, Fan S, Figueiredo AE, et al. ISPD peritonitis recommendations: 2016 update on prevention and treatment. Perit Dial Int 2016; 36(5):481-508

4. Barretti P, Doles JVP, Pinotti DG, El Dib R. Efficacy of antibiotic therapy for peritoneal dialysis-associated peritonitis: a proportional meta-analysis. BMC Infectious Diseases 2014, 14:445

5. Azap OK, Timurkaynak F, Sezer S, CagÁáir U, Yapar G, Arslan H, et al. Value of automatized blood culture systems in the diagnosis of continuous ambulatory peritoneal dialysis peritonitis. Transplant Proc 2006; 38:411-2

6. Piraino B, Bernardini J, Sorkin M. Chemical peritonitis due to intraperitoneal vancomycin. Perit Dial Bull 1986; 7:156-9

7. Smith T, Bailie G, Eisele G. Chemical Peritonitis Associated with Intraperitoneal Vancomycin. DICP. 1991;25(6):602-603.

8. Freiman JP, Graham DJ, Reed TG, McGoodwin EB. Chemical peritonitis following the intraperitoneal administration of vancomycin. Perit Dial Int 1992;12(1):57- 60

9. Wong PN, Mak SK, Lee KF, Fung LH, Wong AK. A prospective study of vancomycin- (Vancoled) induced chemical peritonitis in CAPD patients. Perit Dial Int 1997;17(2):202- 4

10. Newman L, Friedlander M, Tessman M. More Experience With Vancoled Induced Chemical Peritonitis. Perit Dial Int 1990;10(2):182

11. Freitas C, Rodrigues A, Carvalho MJ, Cabrita A. Chemical peritonitis in a patient treated with icodextrin and intraperitoneal vancomycin. Nefrologia 2011; 31(5):625-6

12. Naranjo CA, Busto U, Sellers EM et al. A method for estimating the prob- ability of adverse drug reactions. Clin Pharmacol Ther. 1981; 30:239-45

13. Wang A, Li P, Lai K. Comparation of intraperitoneal administration of two preparations of vancomycin in causing chemical peritonitis. Peri Dial Int 1996; 16:172-4

14. Charney D, Gouge SF. Chemical peritonitis associated with intraperitoneal vancomycin. Am J Kidney Dis 1991;17(1):76-9

15. Rosner MH, Chhatkuli B. Vancomycin-Related Eosinophilic Peritonitis. Perit Dial Int 2010; 30:650-2

16. Deweese R, Slavens J, Barua A, Sutton J. Vancomycin-induced eosinophilic peritonitis. Am J Health-Syst Pharm 2016; 73(9):e244-6

17. Rocklin MA, Teilelbaum I. Noninfectious causes of cloudy peritoneal dialysate. Sem Dial 2001;14(1):37-40

18. Nambiar S, Madurawe RD, Zuk SM, Khan SR, Ellison CD, Faustino PJ. Product quality of parenteral vancomycin products in the United States. Antimicrobial Agents and Chemotherapy 2012; 56(6) 2819-23

\section{Correspondence to:}

José Luís Ceriz Santos Lopes Silvano

Departamento de Nefrologia, Centro Hospitalar de São João, Alameda Prof.

Hernâni Monteiro 4200-319 Porto Portugal

E-mail: jlcsilvano@gmail.com 